\title{
The measure of effectiveness of a short-term 2-week intensive Cardiac Rehabilitation program in decreasing levels of anxiety and depression
}

\author{
Floriana Caccamo1, Simone Saltini2, Enrico Carella2, Roberto Carlon², Cristina Marogna1, \\ Vito Sava2 \\ 1 Department of Philosophy, Sociology, Pedagogy and Applied Psychology, University of Padua \\ 2 UOA of Cardiology, Cardiac Rehabilitation, ASSL 6 Euganea, Presidio Hospital of Cittadella, Padua, Italy
}

\begin{abstract}
Research on heart disease have found a strong and consistent evidence of association between some psychosocial risk factors, including depression, anxiety, self-efficacy, lack of social support and outcome of disease. Depression increases the risk of cardiac death and is highly predictive of reduced adherence to recommended treatments; anxiety appears to be linked to adverse cardiac outcomes. It was demonstrated that Cardiac Rehabilitation (CR) leads to substantial improvements and positive outcomes because combines the prescription of physical activity with the modification of risk factors and aims to reduce symptoms related to the disease and the risk of new cardiovascular events. The main objective of this study is to determine if a short and intense CR
\end{abstract}

Corresponding author: Floriana Caccamo, Department of Philosophy, Sociology, Pedagogy and Applied Psychology, University of Padua, Via Venezia 12, 35124 Padova, Italy.

Tel. +39.320.7244375. E-mail: floriana.caccamo@unipd.it

Key words: Cardiac Rehabilitation; depression; anxiety; effectiveness.

Contributions: CM, FC, VS, RC, study design and data collecting supervision; SS, EC, data collecting: FC, statistical analysis; FC, SS, manuscript drafting. All the authors have critically revised the article and approved the final version.

Conflict of interest: There are no potential conflicts of interest or any financial or personal relationship with other people or organizations that could inappropriately bias conduct and findings of this study.

Acknowledgments: We thank all personnel as well as the care team of the Cardiac Rehabilitation program of Presidio Hospital of Cittadella, particularly doctors, nurses, psychologists, dietitians, physical therapists and trainees, who have welcomed and encouraged the implementation of this study.

Received for publication: 26 May 2017

Accepted for publication: 27 December 2017

CC Copyright F. Caccamo et al., 2018

Tipografia PI-ME Editrice, Italy

Monaldi Archives for Chest Disease 2018; 88:858

doi: 10.4081/monaldi.2018.858

This article is distributed under the terms of the Creative Commons Attribution Noncommercial License (by-nc 4.0) which permits any noncommercial use, distribution, and reproduction in any medium, provided the original author(s) and source are credited. program can produce a positive impact on anxious and depressive symptoms revealed in cardiac patients, confirming results of previous researches. The protocol was proposed to all patients referred for an outpatient CR after an acute event who attended the short 2-week intensive rehabilitation program. A total of 157 patients recruited at the operating unit of Cardiology, in the Hospital of Cittadella (Italy), was included in the analysis. The Beck Depression Inventory-II and the State-Trait Anxiety Inventory-Y were administered to the patients. SPSS 17.0 was used for statistical analysis. T-tests for paired samples were used to evaluate differences between the beginning and the end of the CR program. There was a statistically significant difference between the beginning and the end of the CR program. Results for paired samples showed significant differences in all factors of the BDI-II and in the total score. In addition, a statistically significant difference was found even in the state - anxiety subscale. No significant difference was detected for the trait anxiety. According to recent studies, this research shows that the CR program has a significant impact on levels of anxiety and depression, because all activities focus their commitment on changing the patient's personal beliefs and perception of illness, promoting the exchange of information and sharing of concerns and fears, increasing the patient's resilience with the aim of enabling him/her to reorganize positively his/her personal, family and professional sphere.

\section{Introduction}

Research on heart disease found a strong and consistent evidence of association between some psychosocial risk factors, including depression, anxiety, perception of self-efficacy, lack of social support and outcome of disease [1,2]. Particularly, it was found that depression increases 3 or 4 times the risk of cardiac death and is highly predictive of reduced adherence to recommended treatments after 3 and 12 months $[3,4]$. Anxiety appears to be linked to adverse cardiac outcomes; specifically, it was observed that apprehension is a component of anxiety which increases the probability of developing an adverse cardiac event, as an independent risk factor $[5,6]$. In scientific literature psychological stress is identified as a modifiable risk factor that could facilitate the appearance or even accelerate the aggravation of cardiac disease $[7,8]$.

The results of detailed studies observed that patients with a higher risk of heart problems are people who live in a constant state of emotional tension and anxiety, especially linked to frustration and failure in their social and work activities [9]. Depression and anxiety are common symptoms among patients who have suffered an acute cardiac event and sometimes can persist for months and get to persist for years, substantially impacting patients' quality of life [10,11].

Since anxiety and depression are considered modifiable risk factors, it becomes fundamental the charge of the patient within the Cardiac 
Rehabilitation program (CR), which was demonstrated as an effective treatment that leads to substantial improvements and positive outcomes [12].

Cardiac rehabilitation combines physical activity with risk factors' modification and, within a perspective of secondary prevention, aims to reduce symptoms related to the disease and the risk of new cardiovascular events, to improve the physical exercise capacity, to reduce disability and to improve re-employment [13].

The program is characterized by five fundamental activities. Initially, the patients are subjected to all those cardiological surveys necessary for a better evaluation of its post-acute condition. Secondly, they are led to a period of personal physical training, considering that recent studies found that the introduction of physical activity in a CR program reduces depressive symptoms and cardiovascular mortality and hospitalization rate, resulting in an overall improvement in quality of life $[14,15]$. These studies confirmed that cardiac patients with depressive symptoms benefit of exercise in terms of reduced mortality and cardiovascular comorbidity rates at 12 months away from the acute cardiac [16-19]. The third activity in which patients are involved is health education, with the aim of increasing their resources and self-management skills to change their personal beliefs, sometimes erroneous, to improve compliance and prevent relapses and hospitalizations, to raise awareness of the problem and encourage continuous monitoring, even in subjects with cardiovascular disease but asymptomatic.

In addition, group meetings with a dietitian are provided to start healthy eating programs mainly based on the Mediterranean diet (Food Pyramid of the Mediterranean Diet). Finally, a group of psychological support is provided, since any cardiac event involves a high psychological stress that if prolonged, in addition to worsen the quality of life, may cause an increased risk of cardiovascular events. The group meets once a week and is led by a psychodinamic psychologist. The psychological support group offers to patients a space dedicated to reflection on their own disease, allowing them to share the most difficult anxieties and to understand how to live with the disease [20]. During the group meetings, patients meet people who lived similar experiences and this increases the perception of social support and the reassurance that comes from it [21]. There are also psychological/individual psychotherapeutic talks aimed to the patients who require further investigation, or the patients reported by the nurses. Attention should be given on depressive symptoms since recent studies showed to be an independent risk factor of new cardiac events and mortality, especially in people already suffering from coronary artery disease [3].

Recent studies show the effectiveness of psychological support group treatment in cardiac patients, who can meet common and shared problems, including interpersonal conflicts, life transitions and grief. It also addresses social isolation, a factor that was associated with an increased mortality and morbidity in some studies of patients with postacute or chronic heart disease [22].

The main objective of the present study is to determine whether a short and intense Cardiac Rehabilitation program can produce a positive impact on anxious and depressive symptoms revealed in heart patients, confirming the results of previous research that have largely described the short-term benefits of such rehabilitation program $[23,24]$.

\section{Materials and Methods}

\section{Participants}

Patients were recruited at the operating unit of Cardiology, in the Hospital of Cittadella (Italy). The protocol was proposed to all patients referred for an outpatient CR program after an acute event (ischemic heart disease with or without subsequent myocardial revascularization, chronic heart failure, chronic occlusive peripheral arterial disease, heart transplantation), who attended the short 2-week intensive rehabilitation program (were excluded patients who have an extended program, reserved for the most compromised patients, mainly those with chronic heart failure and patients unable to participate due to family, work or logistic issues). The exclusion criteria concerned the inability to read or understand Italian, the visual or auditory impairments, the incomplete data collection, having life-threatening condition and having a neurological deficit.

This study covered the period from April 2014 to August 2015. Among the 166 patients enrolled, two patients dropped out the rehabilitation program for personal reasons and seven patients were excluded from the analysis because they did not complete the post-test evaluation. Therefore, a total of 157 patients was included in the analysis. Patients were distributed as follows: 125 men (79\%) and 32 women (21\%) with a mean age of $65.2(\mathrm{SD}=9.7)$ years. Compared to diagnosis, patients were as follows: $42 \%$ acute myocardial infarction, $14 \%$ stable angina, $14 \%$ valvular disease, $22 \%$ coronary artery bypass, $8 \%$ dilated cardiomyopathy. All patients gave their written informed consent to participate in this protocol, which was revised and approved by the Ethical Committee of the University of Padua.

\section{Measures}

Patients were administered the Beck Depression Inventory-II (Italian validation of BDI-II) [25] and the State-Trait Anxiety Inventory-Forma Y (Italian validation of STAI-Y) [26].

The BDI-II is a self-report questionnaire widely used to assess the severity of depressive symptoms in the last two weeks preceding the assessment. The questionnaire consists of 21 multiple choice items, of which 13 related to the affective factor (which relates to the somatic and emotional manifestations of depression, such as loss of interest and energy, changes in sleep and appetite, agitation and crying, etc.) and 8 related to cognitive factor (which is primarily concerned with cognitive events such as pessimism, guilt, self-criticism, etc.). Each item is rated on a Likert scale from 0 to 3 and a total score ranging from 0 to 63 is obtained by summing the score of each item. For the analysis of the results it was taken into consideration a cutoff of 16 , as recent Italian studies identified this value as an expression of moderate depressive symptoms $[27,28]$.

The STAI-Y is a self-report questionnaire that allows to determine whether the anxiety present the characteristics of a stable state and persistent emotional (trait anxiety) or rather a temporary condition referring to a time or situation (state anxiety). It consists of 40 items that are rated on a Likert scale from 1 to $4(1=$ not at all and $4=$ very much). Item scores are added to obtain subtest total scores. Range of scores for each subscale is $20-80$, the higher score indicating greater anxiety. A cut point of 39-40 has been suggested to detect clinically significant symptoms for the state-anxiety subscale $[29,30]$.

To verify a change in the levels of anxiety and depression, two administrations were made: the first took place at the beginning of the Cardiac Rehabilitation program (pre-test) and the second was carried out at the end of the rehabilitation (post-test).

\section{Statistical analysis}

Statistical package SPSS 17.0 was used for statistical analysis. Ttests for paired samples were carried out to evaluate differences between pre- and post-evaluation. A p-value of 0.05 or less was considered to indicate statistical significance. 
Both instruments showed good reliability and internal consistency both at pre-test and at post-test. For the BDI-II: $\alpha=0.832$ to pre-test and $\alpha=0.874$ in the post-test. For the STAI-Y: $\alpha=0.886$ at the pre-test and $\alpha=0.861$ at the post-test.

\section{Results}

About depressive symptoms, patients were distributed as follows at the pre-test evaluation: $61 \%$ (minimum depression), 18\% (average depression) and 21\% (severe depression); and at the post-test evaluation: $89 \%$ (minimum depression), 9\% (mean depression) and 2\% (severe depression). With regard to state-anxiety subscale, at the pre-test evaluation a $21 \%$ of patients exceed the cutoff point (39-40], that decreased to $16 \%$ in the post-test evaluation. No patient in the study was following a pharmacological treatment for depression and anxiety.

The t-test results for paired samples showed significant differences in all factors of the BDI-II (affective and cognitive) and in the total score (Table 1); particularly, the average scores decrease between the beginning and the end of the CR program. In addition, a statistically significant difference was found even in the means of state - anxiety subscale, that decrease over time (Table 2). No significant difference was detected for the trait anxiety.

\section{Discussion}

As emerged from the statistical analysis, there was a statistically significant difference between the beginning and the end of the Cardiac Rehabilitation program. This means that the Cardiac Rehabilitation program has a significant impact on the levels of depression and anxiety of patients through the presence of activities that focus on modifying their personal beliefs and perception of illness, promoting information exchange and sharing fears and concerns about their own daily life. The reduction of the prevalence of anxious and depressive symptoms is a significant implication of the study that underlines, as demonstrated in previous studies, the crucial role of rehabilitation in improving the overall quality of life of cardiac patients [14,19]. According to recent studies [18,23], our research shows that the Cardiac Rehabilitation program, although short, is effective in helping patients to understand better the mechanisms underlying their illness and the possibilities of living with it. Through physical activity, health educa- tion, meetings with dietitians and participation in the psychological support group, cardiac patients can feel themselves masters of their own body, increase their awareness of the problem and their resilience, so that they may reorganize positively their personal, family and professional sphere, feeling less anxious and depressed.

It was found that there is no significant reduction of trait anxiety levels: this is an expected result, given that the anxiety component of stretch refers to an individual predisposition, relatively stable over time, which predisposes the patient to perceive situations as threatening and to respond to them with an increase in the level of anxiety. In accordance with this aspect, it would be audacious to expect the change of that component, in fact, relatively stable over time, in a limited treatment period of two weeks.

In the Cardiac Rehabilitation program, there is a whole team dedicated to heart patient care disease, which is responsible to take care of the body's health and psychological well-being and to help patients better understand their disease and the possibilities of treatment. Structured activities within the program have many beneficial effects on the patient's psychological state, starting from the physical activity that, in addition to preventing cardiovascular mortality and morbidity, gives them a more positive image of themselves as people who can be able to perform physical exercises $[14,15]$. In fact, recent studies suggest that physical activity is associated with a better quality of life, as improvements in motor functions, and benefits related to physical activity create a decrease of depressive symptoms [32,33].

Even the psychological support group is an important form of care for patients because it helps them to share their anxieties and concerns associated with the disease. The socialization and interaction created with other patients, who are in different stages of recovery and rehabilitation, are important components of the intervention program and contribute to the favorable effects observed in the levels of anxiety and depression [31]. Many studies confirm the effectiveness of the homogeneous group device for the treatment of physical illnesses, such as heart disease, because it becomes therapeutic for patients to experience understanding and reflection of other patients who have had the same experience and who can provide feedback, reassurance and hope [32,33]. In addition, sharing their problems and difficulties with other patients allows the denial of the unique sensations that many of them may feel [21]: after listening to concerns like their own, patients report a lower sense of isolation and loneliness and a greater acceptance by the other participants.

Although the results of this study emphasize the crucial role of Cardiac Rehabilitation in improving the overall quality of life of cardiac patients,

Table 1. Means and standard deviations of the BDI before (T0) and after (T1) the CR program.

\begin{tabular}{|c|c|c|c|c|c|}
\hline BDI-II & Evaluation & M & SD & $\mathrm{t}$ & p \\
\hline BDI-II - affective factor & $\begin{array}{l}\mathrm{T} 0 \\
\mathrm{~T} 1 \\
\end{array}$ & $\begin{array}{l}5.98 \\
4.98 \\
\end{array}$ & $\begin{array}{l}5.17 \\
4.14 \\
\end{array}$ & 3.38 & 0.001 \\
\hline BDI-II - cognitive factor & $\begin{array}{l}\mathrm{T} 0 \\
\mathrm{~T} 1\end{array}$ & $\begin{array}{l}1.63 \\
0.92 \\
\end{array}$ & $\begin{array}{l}2.48 \\
1.65 \\
\end{array}$ & 4.86 & 0.000 \\
\hline BDI - total score & $\begin{array}{l}\text { T0 } \\
\text { T1 }\end{array}$ & $\begin{array}{l}7.61 \\
5.90 \\
\end{array}$ & $\begin{array}{l}7.13 \\
5.27\end{array}$ & 4.50 & 0.000 \\
\hline
\end{tabular}

Table 2. Means and standard deviations of the STAI-Y before (T0) and after (T1) the CR program.

\begin{tabular}{lccccc} 
STAI-Y & Evaluation & M & SD & t & P \\
STAI-Y - anxiety state & T0 & 35.76 & 8.79 & 2.54 & 0.012 \\
& T1 & 34.32 & 8.02 & & 0.079 \\
\hline STAI-Y - trait anxiety & T0 & 35.62 & 9.40 & 1.77 & 0.00 \\
& T1 & 34.76 & 9.00 & & \\
\hline
\end{tabular}


the study has some limitations. Firstly, the absence of remote follow-up of one year, as suggested by other studies [34], does not support the conclusion that the reduction of depressive and anxious symptoms will be maintained over time. The periodic and structured multidisciplinary follow-up is a tool that allows the continuity of care for cardiac patients.

A further limitation of this study may be due to the selection bias: participation in the cardiac rehabilitation program identifies these patients as early as active patients, who want to take care of themselves and achieve an improvement in quality of life, and therefore distinguishes them from those who have not joined the program or do not have led him to the end. It would be interesting to investigate the reasoning behind these choices, and their relationship with anxious and depressive events detected by the instruments used in this study. Also, the absence of a control group does not consent to generalize results found.

Finally, there were no collections of physical performance objective measures to evaluate possible correlations with psychological symptoms. Other future studies will be oriented to investigate the possible relationship between psychological variables and other symptoms (e.g., disease, social support).

\section{References}

1. Chauvet Gelinier JC, Bonin B. Stress, anxiety and depression in heart disease patients: A major challenge for cardiac rehabilitation. Ann Phys Rehabil Med 2017;60:6-12.

2. Roest AM, Martens EJ, de Jonge P, Denollet J. Anxiety and risk of incident coronary heart disease: a meta-analysis. J Am Coll Cardiol 2010;56:38-46.

3. Huffman JC, Celano CM, Januzzi JL. The relationship between depression, anxiety, and cardiovascular outcomes in patients with acute coronary syndromes. Neuropsych Dis Treat 2010;6:123-36.

4. Abrignani MG, Renda N, Abrignani V, et al. Panic disorder, anxiety, and cardiovascular diseases. Clin Neuropsychiatry 2014;11:130-44.

5. Frasure-Smith N, Lespérance F. Depression and anxiety as predictors of 2-year cardiac events in patients with stable coronary artery disease. Arch Gen Psychiat 2008;6:62-71.

6. Shen BJ, Avivi YE, Todaro JF. Anxiety characteristics independently and prospectively predict myocardial infarction in men: the unique contribution of anxiety among psychologic factors. J Am Coll Cardiol 2008;51:113-9.

7. Bunker SJ, Colquhoun DM, Esler MD, et al. "Stress" and coronary heart disease: psychosocial risk factors. Med J Australia 2003;178:272-6.

8. Glozier N, Tofler GH, Colquhoun DM, et al. Psychosocial risk factors for coronary heart disease. Med J Australia 2013;199:179-80.

9. Neylon A, Canniffe C, Anand S, et al. A global perspective on psychosocial risk factors for cardiovascular disease. Prog Cardiovas Dis 2013;55:574-81.

10. Grace SL, Abbey SE, Irvine J, et al. Prospective examination of anxiety persistence and its relationship to cardiac symptoms and recurrent cardiac events. Psychother Psychosom 2004;73:344-52.

11. Ziegelstein RC, Kim SY, Kao D, et al. Can doctors and nurses recognize depression in patients hospitalized with an acute myocardial infarction in the absence of formal screening? Psychosom Med 2005;67:393-7.

12. Gilbody S, Sheldon T, House A. Screening and case-finding instruments for depression: a meta-analysis. Can Med Assoc J 2008;178:997-1003.

13. World Health Organization. Rehabilitation after cardiovascular diseases, with special emphasis on developing countries: report of a WHO expert committee; 1993.
14. Herring MP, Puetz TW, O'Connor PJ, Dishman RK. Effect of exercise training on depressive symptoms among patients with a chronic illness: a systematic review and meta-analysis of randomized controlled trials. Arch Intern Med 2012;172:101-11.

15. Penedo FJ, Dahn JR. Exercise and well-being: a review of mental and physical health benefits associated with physical activity. Curr Opin Psychiatr 2005;18:189-93.

16. Anderson L, Oldridge N, Thompson DR, et al. Exercise-based cardiac rehabilitation for coronary heart disease: Cochrane systematic review and meta-analysis. J Am Coll Cardiol 2016;67:1-12.

17. Armstrong MJ, Sigal RJ, Arena R, et al. Cardiac rehabilitation completion is associated with reduced mortality in patients with diabetes and coronary artery disease. Diabetologia 2015;58:691-8.

18. Pourafkari L, Ghaffari S, Tajlil A, et al. The impact of cardiac rehabilitation program on anxiety and depression levels after coronary artery bypass graft surgery. Cor et Vasa 2016;58:384-390.

19. Ernstsen L, Rangul V, Nauman J, et al. Protective effect of regular physical activity on depression after myocardial infarction: The HUNT Study. Am J Med 2016; 129:82-8.

20. Da Vico L, Ciompi M, Schininà $\mathrm{F}$, et al. Multidisciplinarietà in riabilitazione cardiologica ed in prevenzione secondaria, dalla valutazione all'educazione terapeutica: un progetto formativo. Monaldi Arch Chest Dis 2014;82:35-42.

21. Yalom I, Leszcz M. Teoria e pratica della psicoterapia di gruppo. Torino: Bollati Boringhieri; 2005.

22. Rutledge T, Redwine LS, Linke SE, Mills, PJ. A meta-analysis of mental health treatments and cardiac rehabilitation for improving clinical outcomes and depression among patients with coronary heart disease. Psychosom Med 2013;75:335-49.

23. Freitas PD, Haida A, Bousquet M, et al. Short-term impact of a 4-week intensive cardiac rehabilitation program on quality of life and anxiety-depression. Ann Phys Rehabil Med 2011;54:132-43.

24. Lavie CJ, Milani RV. Adverse psychological and coronary risk profiles in young patients with coronary artery disease and benefits of formal cardiac rehabilitation. Arch Intern Med 2006;166:1878-83.

25. Ghisi M, Flebus GB, Montano A, et al. Beck Depression Inventory, $2^{\text {nd }}$ edition. Firenze: Organizzazioni Speciali; 2006.

26. Pedrabissi L, Santinello M. [Nuova versione italiana dello STAI forma Y].[in Italian]. Firenze: Organizzazioni Speciali; 1989.

27. Buodo G, Novara C, Ghisi M, Palomba D. Posttraumatic and depressive symptoms in victims of occupational accidents. Depress Res Treat 2012;1-8.

28. Sica $\mathrm{C}$, Ghisi M, Altoè $\mathrm{G}$, et al. The Italian version of the ObsessiveCompulsive Inventory: Its psychometric properties on community and clinical samples. J Anxiety Disord 2009;23:204-11.

29. Knight RG, Waal-Manning HJ, Spears GF. Some norms and reliability data for the State-Trait Anxiety Inventory and the Zung SelfRating Depression scale. Br J Clin Psychol 1983;22:245-9.

30. Addolorato G, Ancona C, Capristo E, et al. State and trait anxiety in women affected by allergic and vasomotor rhinitis. J Psychosom Res 1999;46:283-9.

31. Corbella S, Girelli R, Marinelli S. Gruppi omogenei. Milano: Borla; 2001.

32. Whalley B, Thompson DR, Taylor RS. Psychological interventions for coronary heart disease: cochrane systematic review and metaanalysis. Int J Behav Med 2014;21:109-21.

33. Griffo R, Urbinati S, Giannuzzi P, et al. [Italian guidelines on cardiac rehabilitation and secondary prevention of cardiovascular disease: executive summary].[Article in Italian]. G Ital Cardiol (Rome) 2008;9:286-97.

34. Kavanagh T, Mertens DJ, Hamm LF, et al. Prediction of long-term prognosis in 12169 men referred for cardiac rehabilitation. Circulation 2002;106:666-71. 